\title{
Review
}

\section{Autofluorescence endoscopy for the gastrointestinal tract}

\author{
By Hisao TAJIRI ${ }^{* 1, \dagger}$ \\ (Communicated by Takashi Sugimura, M.J.A.)
}

\begin{abstract}
This review focuses on our basic study results and clinical experience of fluorescence endoscopy for the gastrointestinal (GI) tract. Collagen, which fluoresces in the green wavelength range, is one of the major sources of tissue autofluorescence (AF) and AF imaging systems are now available. With their use, however, it is important to take into account tissue changes other than, or in addition to, changes in gross tissue morphology. These may include alterations in the local blood volume, tissue metabolic activity, and relative fluorophore concentrations. New AF imaging systems are very easy to use, because white light endoscopy can be changed to AF at the push of a button, and hold great promise for diagnosis of early carcinomas and premalignant lesions in the GI tract. In particular, AF endoscopy has potential for identification of small or flat tumors, tumor margins and premalignant lesions in Barrett's esophagus, as well as for assessing tumor grade and response to therapy. However, large-scale studies are needed to clarify the clinical impact of this new diagnostic approach.
\end{abstract}

Keywords: Gastrointestinal tract, autofluorescence endoscopy, light-induced fluorescence endoscopy, white light endoscopy, charge-coupled device, Barrett's esophagus

\section{Introduction}

Fluorescence endoscopy to detect early carcinomas and to discriminate between normal and neoplastic lesions has recently attracted considerable attention. Spectroscopic techniques can be classified mainly into two types: photodynamic diagnosis using fluorescence drugs called photosensitizers that react to various wavelengths of light, ${ }^{1,2)}$ and autofluorescence (AF) diagnosis, employing inherent tissue AF without the use of additional photosensitizers. To detect AF differences as endoscopic images has been very difficult,

*1 Department of Gastroenterology and Hepatology, The Jikei University School of Medicine, Tokyo, Japan.

$\dagger$ Correspondence should be addressed: H. Tajiri, M.D. Department of Gastroenterology and Hepatology, The Jikei University School of Medicine, 25-8, Nishishinbashi 3-chome, Minato-ku, Tokyo 105-8461, Japan (e-mail: tajiri@jikei.ac.jp).

Abbreviations: GI tract: Gastrointestinal tract; AF: autofluorescence; AFI: autofluorescence imaging system; LIFE: lightinduced fluorescence endoscopy; FMN: flavin mononucleotide; FAD: flavin adenine dinucleotide; NADH: reduced nicotinamide adenine dinucleotide; BE: Barrett's esophagus; CCD: chargecoupled device; RGB: red, green and blue; WLE: white light endoscopy; ESD: endoscopic submucosal dissection; NBI: narrow band imaging. as AF can be too faint to detect. However, the development of powerful light sources and high sensitivity cameras has led to major advances in AF imaging systems, such as the light-induced fluorescence endoscopy in the gastrointestinal (GI) tract system (LIFE-GI system; Xillix Technology Co., Canada) or the lung imaging or laser induced fluorescence endoscopy system (LIFE system; Xillix Technology Co.) for imaging green and red fluorescence in real time. These systems have been applied clinically and found to be very useful. ${ }^{3), 4)}$

Since prior AF imaging systems using fiberoptic endoscopes and heavy image intensifying camera units attached to eyepiece failed to provide sufficient image quality and maneuverability, they were not appropriate for general clinical use in the era of high resolution videoendoscopy. In addition, because both tumors and hemoglobin absorb AF, use of AF alone resulted in low specificity in the LIFE system. Therefore, a novel videoendoscopy system using a combination of $\mathrm{AF}$ and reflectance imaging (Olympus Medical Systems, Co., Tokyo, Japan) was developed. This review focuses on our basic study results and clinical experience of fluorescence endoscopy in the GI tract. 


\section{Mechanisms of autofluorescence endoscopy}

A number of biological fluorophores, such as flavins (e.g. riboflavin, flavin mononucleotide [FMN] and flavin adenine dinucleotide [FAD]), reduced nicotinamide adenine dinucleotide (NADH), collagen and pyridoxal 5'-phosphate have been reported as potentially responsible for observed differences in AF spectra between normal and diseased tissues. Our studies showed that the main source of tissue fluorescence is submucosal collagen. ${ }^{5}$ The masking effect of thickened mucosa overlaying the submucosal layer is also an important factor in reduced fluorescence of diseased tissues. In other words, in the presence of adenomatous or cancerous proliferation, abnormal epithelium is thicker than normal epithelium, and fluorescence-exciting light from lasers or lamps using special filters may be blocked or reabsorbed to a greater degree than with normal epithelium. On the other hand, the increased blood volume in diseased tissues has also been proposed as an alternative explanation of differences in fluorescence, because hemoglobin has an absorption effect in the green spectrum. DaCosta et al. have proposed that LIFE imaging in vivo can: (i) differentiate between hyperplastic and adenomatous polyps of the same gross size and shape; (ii) detect flat adenomas that have no gross mucosal thickening and are topographically similar to surrounding mucosa; (iii) delineate the boundary of Barrett's esophagus (BE); and (iv) in some cases, enable detection of dysplastic lesions in a Barrett's field. ${ }^{6)}$ This suggests that there are important tissue changes other than, or in addition to, changes in gross tissue morphology. These may include alterations in the local blood volume, tissue metabolic activity, and relative fluorophore concentrations (Fig. 1).

Collagen, which fluoresces in the green wavelength range, is one of the major sources of tissue AF. In a subanalysis of malignant lesions from patients who underwent gastrectomy or mucosectomy, this was demonstrated by Mayinger B. et al. ${ }^{7)}$ Staining of all tissue layers in resection specimens for collagen demonstrated that two thirds of the spectra recorded from gastric carcinomas containing signet-ring cells with proven moderate or massive collagen concentration were false-negatives, whereas this was the case for only $22 \%$ of spectra from adenocarcinomas with a confirmed low

\section{Attenuation Mechanism of Auto Fluorescence}

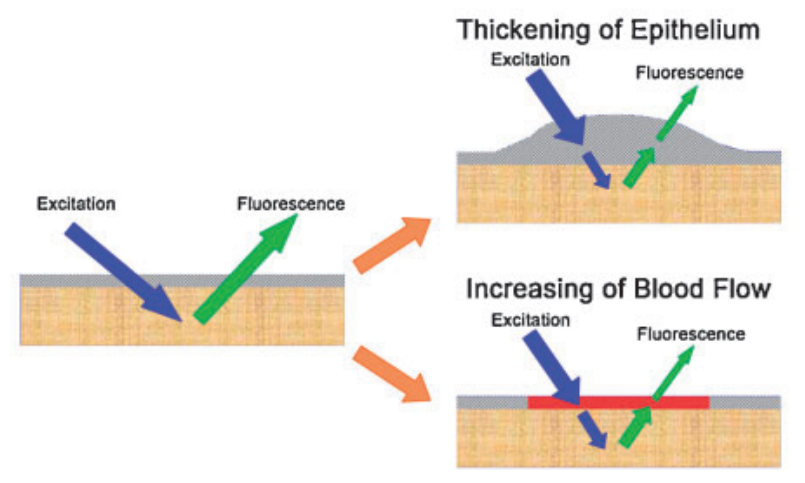

Fig. 1. Attenuation mechanism underlying detection of lesions by autofluorescence. On the left are panels depicting normal mucosa, and on the right is a pattern for a tumor. The excitation light is absorbed and scattered in the tumor by the thickening of the mucosa. Therefore, it does not reach fluorescent material that exists in the submucosal layer, and excited autofluorescence is consequently diminished. Moreover, the blood flow volume to tumors is more abundant than to normal mucosa. The hemoglobin contained in blood has the characteristic of absorbing blue light strongly. Therefore, the amount of light impacting on fluorescent material is reduced and autofluorescence decreases.

concentration of collagen. Severe arophy of tumorous mucosa would appear to be an additional risk factor for incorrect-negative AF spectra.

\section{Clinical methods for autofluorescence endoscopy}

We have been using the LIFE-GI system since 1996, this being composed of a mercury lamp and a blue excitation filter, white light source, two highsensitivity charge-coupled device (CCD) cameras, a color CCD camera for white light, an endoscope, and a monitor. First, $437 \mathrm{~nm}$-wavelength blue light from the mercury lamp is filtered through the excitation filter and is used to illuminate the tissue through the endoscope. The AF generated is then amplified by the high sensitivity CCD camera attached to the end of the endoscope. This camera has two built-in high sensitivity imaging elements that capture green (wavelength: 490-560 nm) and red (wavelength: >630) AF. After the individual image signals are processed by the image processor in real time, they are displayed on the monitor in pseudo-color. The cancerous areas are displayed as dark red and the healthy areas as light blue. Use of a metal-halide lamp as a light source has allowed much clearer and brighter images to be obtained since 1998 . 
For AF endoscopy, a new AF imaging system (AFI, Olympus Medical Systems Co.) has been available since 2001. While the basic configuration is identical to the video-endoscopy system (LUCERA CV-260/CLV-260, Olympus Medical Systems Co.), the new AFI system features a switch for selecting either Red, Green, and Blue (RGB) illumination light for normal imaging (white light endoscopy, WLE) or a excitation/reflected light illumination light combination for AFI. The light source incorporates a rotary filter, which is designed in a double-wheel configuration with two concentric wheels: an RGB filter wheel for normal imaging and an AFI filter wheel. When the AFI mode is selected, the light emitted from the xenon lamp is input to the rotary filter and divided into the 390 to $470 \mathrm{~nm}$ excitation light and 540 to $560 \mathrm{~nm}$ green light. The AFI scope (XGIF-Q240FZ, Olympus Medical Systems Co.) incorporates a monochrome CCD that has a barrier filter for cutting the excitation light to capture the weak AF. A pseudo-color image is reconstructed based on the AF input signals so that high AF intensity is colored as greenish, and low intensity as magenta (Fig. 2). In general, the AFI system denotes normal pyloric mucosa or atrophic fundic mucosa in green, whereas hypertrophic fundic mucosa or elevated neoplasias are magenta and blood vessels are dark green.

\section{Clinical application of autofluorescence endoscopy}

1. Gastric cancer lesions. A comparative assessment of fluorescence images and histopathological findings was conducted to clarify what colors are seen in gastric cancers with the LIFE-GI system. ${ }^{8)}$ A total of 429 evaluation points in specimens from 61 gastric cancer were selected according to the tumor size. Detection with this system was possible in $58(95.1 \%)$ of the 61 cases, and most of the cancer areas appeared dark red. The detection rates increased with the depth of invasion and also increased significantly as the mucosa that was invaded became thicker. Therefore, mucosal thickening as a result of cancer cell invasion had a large impact on the detection rate with this system.

We then applied the LIFE-GI system to patients with early gastric cancers to investigate its clinical utility. ${ }^{9)}$ From comparisons of AF images and histology, 21 of 33 carcinomas appeared dark red, 10 demonstrated a mixed pattern of dark red

\section{System Configuration}

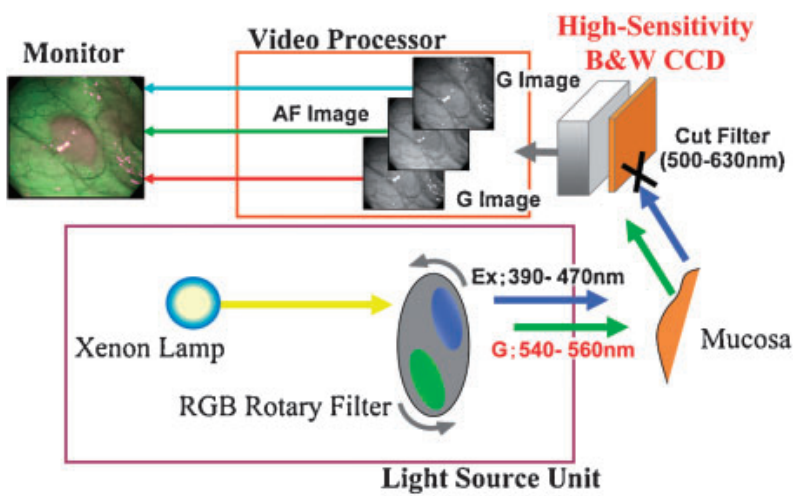

Fig. 2. AFI sequential method. The light source incorporates a rotary filter, which is designed in a double-wheel configuration with two concentric wheels: an RGB filter wheel for normal imaging and an AFI filter wheel. When the AFI mode is selected, the light emitted from the xenon lamp is input to the rotary filter and divided into 390 to $470 \mathrm{~nm}$ excitation light and green light. The AFI scope incorporates a monochrome CCD that has a barrier filter for cutting the excitation light to capture the weak AF. A pseudo-color image is reconstructed based on the AF input signals so that high AF intensity is colored as greenish, and low intensity as magenta.

and white fluorescence, and the remaining two could not be detected. Furthermore, the cancer extension recognized by this system corresponded well to that confirmed by the histology in $85 \%$ $(28 / 33)$ of cases. On the other hand, 18 of 21 benign lesions appeared light blue, similar to normal mucosa, while the others showed reddish color. The sensitivity and specificity were $94 \%$ and $86 \%$, respectively. In a study by Ogihara et al. all of 20 gastric cancers were observed as dark red, three of five gastric adenomas were light red, and the remaining two were dark red. ${ }^{10)}$ This system has also been applied for the detection of remnant lesions after endoscopic treatment of early gastric cancer. Remnant tumors were suspected in four of 12 cases using the LIFE-GI system, two of which were found by histological methods to be adenocarcinoma but were not detected by conventional WLE. The results suggest that small neoplastic lesions, which are difficult to diagnose by WLE, can be detected by the system.

Recently, to clarify relevance, we conducted a prospective study comparing the new AFI with conventional WLE for the detection of early gastric cancers. ${ }^{11)}$ Thirty-three patients referred for endo- 

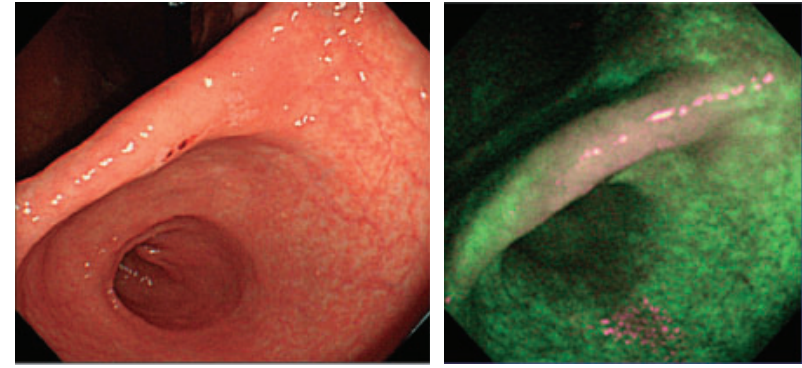

Fig. 3. A representative case with flat elevated type early gastric cancer. In the left panel, a flat elevated lesion of $25 \mathrm{~mm}$ diameter is depicted by WLE. On the right, the same lesions shows magenta color in AFI, and could be diagnosed as neoplastic.

scopic submucosal dissection (ESD), and 18 cancerfree patients with a past history of ESD were enrolled. In the series serially performed by one endoscopist, $13 \%$ of all neoplasias and $23 \%$ of elevated neoplasias were missed by WLE, but detected by AFI. Thus the modality promises clinical relevance with further refinement for improving diagnostic specificity. The AF endoscopy applied was the new AFI featuring video-endoscopy, which has advantages over AFI by fiberscopy (Fig. 3). However, the AF images are coarse in comparison with standard WLE because AF itself is very weak, and the signal to noise ration is still low in the AFI system. If limitations of the current AFI model can be overcome, subtle differences in AF endoscopy color can be enhanced, with consequent increase in diagnostic sensitivity and improvement in detection of early gastric cancer. In principle, however, high false AF-positive rates induced by non-neoplastic protrusions remain to be overcome. Therefore, a combination with a modality to reduce false AF endoscopy-positives may be essential, and the narrow band imaging (NBI) system ${ }^{12), 13)}$ needs to be tested for this purpose as a high priority.

2. Colorectal lesions. AF endoscopy has been also studied clinically in the colon. Cases with ninety-four colorectal lesions, comprising 12 hyperplastic polyps, 6 colon submucosal tumors (SMT), 33 of tubular adenomas and 43 early colon cancers, excised endoscopically or surgically, were enrolled. ${ }^{14)}$ All 76 epithelial neoplastic lesions were observed to have a magenta color whereas the normal background mucosa was green (Fig. 4, 5, 6). All 18 non-neoplastic hyperplastic polyps and SMTs demonstrated the same color as surrounding

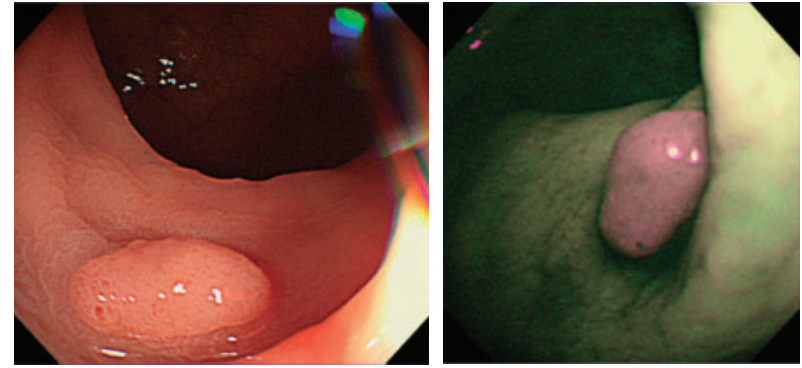

Fig. 4. Endoscopic images of an adenoma of the colon with WLE and AFI. A reddish polyp of $10 \mathrm{~mm}$ in size could be recognized by WLE. On the right, the AFI of the same polyp shows magenta color. Histologically, the polyp was diagnosed as an adenoma with moderate dysplasia.
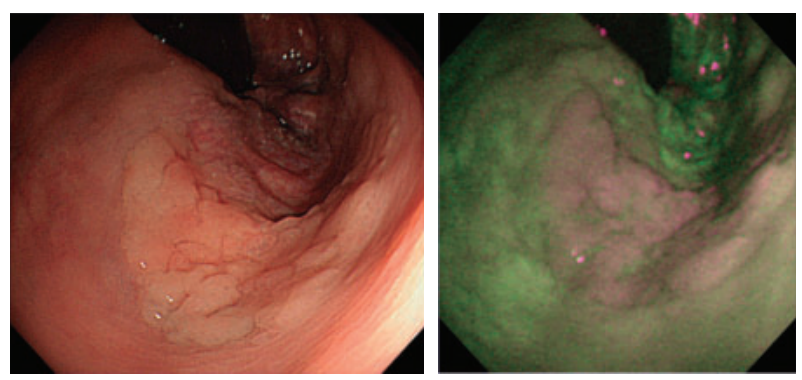

Fig. 5. Endoscopic images of a laterally spreading tumor with granular change of the rectum with WLE and AFI. On the right, AFI shows a magenta color for the lesion, whose boundary can be recognized more clearly than the image with WLE. Histologically, the lesion was diagnosed as an adenoma with severe dysplasia after endoscopic mucosal resection.

mucosa. The sensitivity and specificity for diagnosis of neoplastic lesion were 93\%/94\% for WLE and $97 \% / 100 \%$ for AF endoscopy, and the difference was not significant. However, the boundaries of neoplastic lesions, especially of flat elevated type, could be recognized more clearly than with WLE. Thus utility for differential diagnosis of epithelial neoplasia and non-neoplasia was indicated, especially for adenomatous lesions and hyperplastic polyps, because of the change to magenta color.

3. Esophageal lesions. In the esophagus, the main aim is to detect and discriminate between lowgrade and high-grade dysplasia and cancerous lesions within Barrett's esophagus (BE). In Japan, there the incidence of adenocarcinomas in $\mathrm{BE}$ remains low, and the detection of superficial esophageal squamous cell carcinoma, which is limited to the mucosa or submucosa, has increased markedly because of recent progress in diagnostic techniques, 

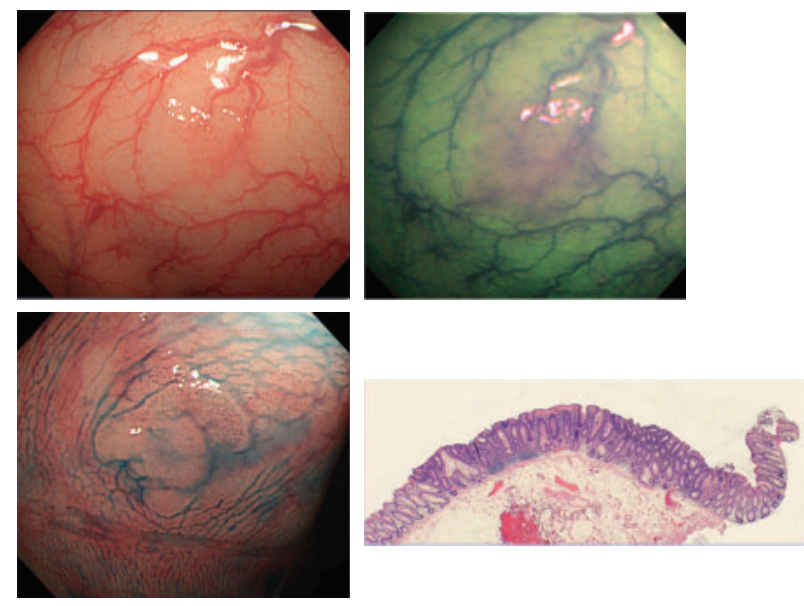

Fig. 6. A case with a flat elevated lesion (adenoma) of the colon. The upper left panel illustrates a flat elevated lesion of $10 \mathrm{~mm}$ in size, and a lower panel shows an indigocarminespraying endoscopic image with WLE. At the upper right, an endoscopic image with AFI reveals a magenta color, and the margin of the lesion can be clearly recognized, as with the dye-spraying endscopic image. Histologically, the lesion was diagnosed as an adenoma (lower right).

especially electronic endoscopy. Several research groups in Europe and North America have reported sensitivities and specificities for AF endoscopy of more than $80 \%$ for carcinoma of the esophagus. Panjehpour et al. analyzed spectra using a method based on the fluorescence intensity at $480 \mathrm{~nm}$ compared to the fluorescence signal integrated over all wavelengths in 36 patients with BE. 15) The classification was considered accurate for $96 \%$ of nondysplastic BE, $100 \%$ of low-grade dysplasias and $90 \%$ of high-grade dysplasias. This demonstrated the potential to identify endoscopicallyoccult premalignant lesions by AF endoscopy.

Kara et al. ${ }^{16)}$ evaluated sixty patients with BE using WLE and AF endoscopy. A diagnosis of highgrade dysplasia (HGD)/early cancer (EC) was made in 22 patients, and 21 had endoscopically detectable areas with $\mathrm{HGD} / \mathrm{EC}$. In 6 of the latter 21, the $\mathrm{HGD} / \mathrm{EC}$ was detected with AFI alone; in another patient, HGD/EC was detected with AFI and random biopsies. In 14 patients, HGD/EC was detected with both WLE and AFI; in 3 of these, additional HGD/EC lesions were detected by AFI alone. The results thus indicated that AFI may improve the detection of $\mathrm{HGD} / \mathrm{EC}$ in $\mathrm{BE}$ patients. Uedo et $a l .{ }^{17)}$ reported two of 5 superficial esophageal cancers $(40 \%)$ to be correctly diagnosed by
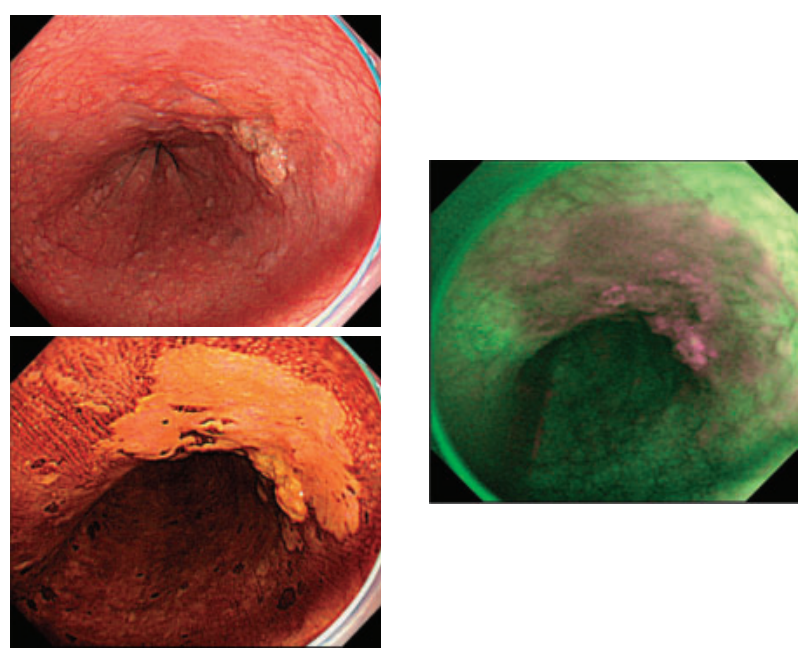

Fig. 7. A case with squamous cell carcinoma of the esophagus. In the upper panel on the left, WLE shows a shallow depressed lesion with granular changes. Endoscopic findings with iodine staining are illustrated on the lower left and the endoscopic image with AFI, showing a magenta color, on the right.

WLE and all (100\%) by AF endoscopy as having a purple or magenta color on a green background. AF endoscopy could reveal flat or isochromatic extensions that were not detected by WLE.

We have also studied the utility of AF endoscopy in screening for early detection of squamous cell carcinoma (SCC) of the esophagus (unpublished data). Twenty cases of superficial SCC of the esophagus, resected endoscopically or surgically between September 2005 and October 2006, were enrolled. It was clarified that AF endoscopy might be useful when endoscopists are less than 35 years old with less than 10 years endoscopic experience, although there was no generally significant difference in diagnostic ability between WLE and AF endoscopy (Fig. 7).

4. Pancreato-biliary cancer lesions. When nine patients with bile duct cancer underwent percutaneous transhepatic AF cholangioscopy using the LIFE-GI system, ${ }^{18}$ all cancerous lesions were observed to be dark red in color. Additionally, in seven out of the nine cases, white fluorescence was also seen in the malignancy. Examination of resected specimens showed the white regions to correspond to area where abundant fibrous stroma was exposed to the internal lumen in the cancerous lesion. Bile duct cancer is well known to feature abundant desmoplastic stroma and white fluorescence might indicate regions where changes are 
due to cancer infiltration. In addition, in a first case report, we described observation of a pancreas cancer with an AFI system. ${ }^{19)}$ In the pancreatic duct, the cancer was also dark red.

\section{The promise of autofluorescence endoscopy for identification of early carcinomas and precancerous lesions}

Efforts are ongoing to develop and evaluate fluorescence-based diagnostic technologies for the detection of premalignant and early stage malignant lesions in many organs. These exploit either naturally occurring autofluorescence from endogenous fluorophores, or fluorescence caused by an exogenously administered fluorescent drug, e.g., 5aminolevulinic acid. With exogenous fluorophores, the detection of the lesion is based on selective drug uptake or retention by the target tissue relative to that by normal tissue. In our studies, only AF emitted from endogenous fluorophores was considered. The AFI used is the first imaging system capable of detecting endogenous tissue AF by incorporating a CCD into the videoendoscope. The resolution is lower than with WLE but the image quality is better than with prior fiberoptic AF endoscopy (LIFE-GI system) for clinical cases. The development of new CCDs and image processing is responsible for such improvement. A comparison of the video and the fiber-optic systems in terms of imaging performance is shown in Table 1. ${ }^{20)}$ Advantages of the former include the following: (1) the resolution elements are determined by the number of pixels in the CCD detector, which is in the order of 100,000 , whereas that of fiber optics is approximately 10,000, a factor of 10 times; (2) videos detect fluorescence photons immediately after the objective lens to achieve a good signal-to-noise ratio (SNR), whereas fiber optics

Table 1. A comparison of imaging parameters between video and fiber-optic systems for performing autofluorescence imaging

\begin{tabular}{lll}
\hline Image parameter & Video & Fiber-optic \\
\hline Resolution elements & $\sim 100,000$ & $\sim 10,000$ \\
Intensifier & No & Yes \\
Contrast & Higher & Lower \\
Registration & Complete & Incomplete \\
Spectral filtering & Fixed & Flexible \\
\hline
\end{tabular}

Source: Wang, T.D., and Triadafilopoulos, G. (2005) Gastrointest. Endosc. 61, 686-688. transmit these photons through an inefficient bundle and require an expensive image intensifier to provide an adequate SNR; (3) the intrinsic contrast for video is higher than with fiber optics, whose larger resolution elements detract from the contrast; and (4) with video instrument, both white light and AF images can detected with the same $\mathrm{CCD}$, resulting in complete image registration; in contrast, with a fiber-optic system, a separate CCD must be used to collect the white-light images.

In conclusion, AFI is very easy to use, because change from WL to AF can be achieved at the push of a button. As detailed above, there is already considerable evidence that AF endoscopy has promise for diagnosis of early carcinomas and premalignant lesions in the GI tract as an adjunct to conventional WLE. In particular, it has good potential for identifying small or flat tumors and tumor margins, as well as premalignant lesions of $\mathrm{BE}$, and for assessing grade and tumor response to therapy. However, further large-scale studies should now be performed to clarify the clinical impact of AF endoscopy. Especially, relationships between AF images and changes in background mucosa such as gastric atrophy or metaplasia need to be established.

\section{References}

1) Kato, H., Imaizumi, T., Aizawa, K., Iwabuchi, H., Yamamoto, H., Ikeda, N., Tsuchida, T., Tamachi, Y., Ito, T. and Hayata, Y. (1990) Photodynamic diagnosis in respiratory tract malignancy using an excimer dye laser system. J. Photochem Photobiol. 6, 189-196.

2) Tajiri, H., Yokoyama, K., Boku, N., Ohtsu, A., Fujii, T., Yoshida, S., Sato, T., Hakamata, K., Hayashi, K. and Sakata, I. (1997) Fluorescent diagnosis of experimental gastric cancer using a tumor-localizing photosensitizer. Cancer Lett. 111, 215-220.

3) Palcic, B., Lam, S., Hung, J. and MacAulay, C. (1991) Detection and localization of early lung cancer by imaging techniques. Chest 99, 742743.

4) Stepp, H., Sroka, R. and Baumgartner, R. (1998) Fluorescence endoscopy of gastrointestinal diseases: basic principles, techniques, and clinical experience. Endoscopy 30, 379-386.

5) Izuishi, K., Tajiri, H., Fujii, T., Boku, N., Ohtsu, A., Ohnishi, T., Ryu, M., Kinoshita, T. and Yoshida, S. (1999) The histological basis of detection of adenoma and cancer in the colon by autofluorescence endoscopic imaging. Endoscopy 31, 511-516.

6) DaCosta, R.S., Wilson, B.C. and Marcon, N.E. 
(1999) Recent advances in light-induced fluorescence endoscopy (LIFE) of the gastrointestinal tract. Dig. Endosc. 11, 108-118.

7) Mayinger, B., Jordan, M., Horbach, T., Horner, P., Gerlach, C., Mueller, S., Hohenberger, W. and Hahn, E.G. (2004) Evaluation of in vivo endoscopic autofluorescence spectroscopy in gastric cancer. Gastrointest. Endosc. 59, 191-198.

8) Abe, S., Izuishi, K., Tajiri, H., Kinoshita, T. and Matsuoka, T. (2000) Correlation of in vitro fluorescence endoscopy images with histopathological findings in stomach cancer. Endoscopy 32, $1-6$.

9) Kobayashi, M., Tajiri, H., Seike, E., Shitaya, M., Tounou, S., Mine, M. and Oba, K. (2001) Detection of early gastric cancer by a real-time autofluorescence imaging system. Cancer Lett. 165, 155-159.

10) Ogihara, T., Watanabe, H., Namihisa, A., Kobayashi, O., Miwa, H. and Sato, N. (1999) Clinical experience using a real time autofluorescence endoscopy system in the gastrointestinal tract. Diagnostic and Therapeutic Endoscopy 5, 119-124.

11) Kato, M., Kaise, M., Yonezawa, J., Yoshida, Y., Yamasaki, T., Imazu, H. and Tajiri, H. A prospective study comparing autofluorescence endoscopy with conventional endoscopy for the detection of superficial gastric neoplasm. Endoscopy (in press).

12) Tajiri, H., Matsuda, K. and Fujisaki, J. (2002) What can we see with the endoscopy? Present status and future perspectives. Dig. Endosc. 14, 131-137.

13) Nakayoshi, T., Tajiri, H., Matsuda, K., Kaise, M., Ikegami, M. and Sasaki, H. (2004) Magnifying endoscopy combined with narrow band imaging system for early gastric cancer: correlation of vascular pattern with histopathology. Endoscopy
36, 1080-1084.

14) Saito, S., Mashiko, T., Imazu, H., Arakawa, H., Kaise, M., Tajiri, H., Ikegami, M. and Tsuruta, O. (2007) Novel autofluorescence imaging system is useful for detection of neoplastic lesion in colon tumor. Gastrointest. Endosc. 65, AB342. (abstract).

15) Panjehpour, M., Overholt, B. F., Vo-Dinh, T., Haggitt, R. C., Edwards, D. H. and Buckley, F. P. 3rd. (1996) Endoscopic fluorescence detection of high-grade dysplasia in Barrett's esophagus. Gastroenterology 111, 93-101.

16) Kara, M. A., Peters, F. P., Kate, F. J. W., van Deventer, S. J., Fockens, P. and Bergman, J. J. G. H. M. (2005) Endosocpic video autofluorescence imaging may improve the detection of early neoplasia in patients with Barrett's esophagus. Gastrointest. Endosc. 61, 679-685.

17) Uedo, N., Iishi, H., Tatsuta, M., Yamada, T., Ogiyama, H., Imanaka, K., Sugimoto, N., Higashino, K., Ishihara, R., Narahara, H. et al. (2005) A novel videoendoscopy system by using autofluorescence and reflectance imaging for diagnosis of esophagogastric cancers. Gastrointest. Endosc. 62, 521-528.

18) Izuishi, K., Tajiri, H., Ryu, M., Furuse, J., Maru, Y., Inoue, K., Konishi, M. and Kinoshita, T. (1999) Detection of bile duct cancer by autofluorescence cholangioscopy: a pilot study. Hepato-Gastroenterol. 46, 804-807.

19) Izuishi, K., Furuse, J., Kinoshita, T., Konishi, M. and Tajiri, H. (2003) Autofluorescence endoscopy images of pancreas cancer: report of a case. Hepato-Gastroenterol. 50, 366-367.

20) Wang, T.D. and Triadafilopoulos, G. (2005) Autofluorescence imaging: have we finally seen the light? Gastrointest. Endosc. 61, 686-688.

(Received June 4, 2007; accepted July 13, 2007)

\section{Profile}

Hisao Tajiri was born in 1949. After graduating from Hokkaido University School of Medicine in Sapporo, Japan, in 1976, he received postgraduate professional training at the Department of Internal Medicine at Cancer Institute Hospital and the Endoscopy Division at National Cancer Center Hospital in Tokyo. He performed research work on experimental pancreatic carcinoma using golden hamsters, laser hyperthermia and photodynamic therapy using tumor-localizing photosensitizers at National Cancer Center between 1981 and 1990. In 1990, Tajiri was appointed Assistant Professor of the Second Department of Internal Medicine at National Defense Medical College, and in 1995, he joined National Cancer Center Hospital East as the Chief of the Endoscopy Division. He was promoted to Professor in the

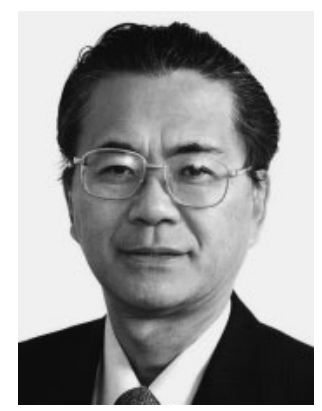
Department of Endoscopy at the Jikei University School of Medicine, Tokyo, in 2001 and became Chairman and Professor in the Division of Gastroenterology and Hepatology, Department of Internal Medicine at the Jikei University School of Medicine in 2005, where he has educated many students and residents in the field of Gastroenterology. For more than 2 decades, he has continued basic and clinical study on image-enhanced 
endoscopy such as fluorescence endoscopy, narrow band imaging, endoscopic microscopy and so on.

Tajiri was awarded the Japanese Cancer Association Award in 1987, the Japan Pancreas Society Award in 1991, and Japan Gastroenterological Endoscopy Society Award in 1994. He received "Crystal Award" from American Society for Gastrointestinal Endoscopy (ASGE) in 2007 and was elected an honorary member of ASGE. He has been active as a member of the Education and Training Committee of the World Gastroenterology Organization. In addition, he has been organizing "Endoscopy Forum Japan" in his role as chairman since 2004, in order to make progress in multi-center randomized prospective studies, and also academic and industrial collaboration in endoscopic medicine. 